\title{
On the Construction of Biobank in General Hospitals
}

\author{
Meng Yu ${ }^{1, a}$, Chen Du ${ }^{2, a}$, Qingyu Yan ${ }^{1}$, Chi Zhang ${ }^{1}$, Feixiang Wu ${ }^{1}$, Cheng $\mathrm{Zhou}^{1 *}$ \\ ${ }^{1}$ Affiliated Hospital of Hebei University, Baoding 071000, China. E-mail: 3154599585 @qq.com \\ ${ }^{2}$ Baoding Children's Hospital, Baoding 071000, China. E-mail: 393250949@qq.com
}

\begin{abstract}
Objective: Discussion on a series of biospecimen related issues are conducted, such as collection and preservation, quality control, as well as management and application, during the construction of human tissue biobank in a general hospital. Methods: To develop a set of standardized operational procedures and to collect tissue samples, such as whole blood, serum, plasma, fresh frozen tissues, cerebrospinal fluid, and paraffin-embedded tissues, which were classified and made aliquots according to different requirements, and stored at $-80^{\circ} \mathrm{C}$ temperature refrigerator or in liquid nitrogen. At the same time, a set of information management software was used to realize management of the biobank. Results: Currently, there are more than 20,000 specimens of various benign and malignant cases, which cover 380 diseases, being collected in the biological database in our hospital. These specimens include paraffin-embedded tissue, fresh frozen tissue, femoral head, whole blood, plasma, serum and cerebrospinal fluid, etc. A large number of these specimens are beneficial is used in clinical research at present. Conclusion: The establishment of biological sample bank can maximize the value of non-reborn human tissue specimens, and provide normal control standards as well as benign and malignant disease standards for clinical diagnosis and treatment, which is of great significance to the research of disease pathogenesis and the development of detection technology.
\end{abstract}

Keywords: General Hospital; Biobank; Hospital Research

Biobank refers to the standardized collection, processing, storage and application of biospecimen, such as cells, tissues, macromolecules and organs of diseases and healthy organisms, etc., as well as corresponding pathological, clinical, therapeutic, follow-up, quality control and information management application system. Biobank is the foundation of medical research and translational medicine with a critical position in disease diagnosis, pathological research, prevention and individualized treatment ${ }^{[1]}$. In recent years, biobanks have been set up one after another in China's major general hospitals with a rapid growing scale and quantity. The biobank in our hospital was founded in 2010, and it has now basi- cally formed a standardized system of sample collection, storage and management. This study analyzes the construction and management of biobank in general hospitals and discusses the ethical review problems faced by the current biobanks as follows.

\section{Materials and methods}

\subsection{Equipment}

Facilities include two specimen storage rooms for placing freezers, refrigerators, liquid nitrogen tanks and other refrigeration equipment. The storage room is e-

a: These authors contributed equally to this work.

Copyright (C) 2020 Meng Yu et al.

doi: 10.18686/aem.v9i3.171

This is an open-access article distributed under the terms of the Creative Commons Attribution Non-Commercial License

(http://creativecommons.org/licenses/by-nc/4.0/), which permits unrestricted non-commercial use, distribution, and reproduction in any medium,

provided the original work is properly cited. 
quipped with $-80^{\circ} \mathrm{C}$ temperature refrigerators, high-efficiency vapor-phase liquid nitrogen storage systems, $-30^{\circ} \mathrm{C}$ refrigerator, $4^{\circ} \mathrm{C}$ refrigerator, centrifuge, barcode printer and scanner. The sampling room is equipped with sampling console, computers, digital cameras, printers, liquid nitrogen tanks, etc. The experimental equipment in the tissue bank includes electrothermal constant temperature blast drying box, constant temperature water bath box, ultraviolet lamp, acidimeter, electrophoresis instrument, tissue chip instrument, vortex mixer, microscope, ultraviolet spectrophotometer, purification workbench, secondary embedding instrument, constant temperature stirrer and so on.

\subsection{Sampling specimen}

\subsubsection{Fresh tissue specimen}

Firstly, one needs to print out the operation schedule of the day in order to collect the isolated surgical specimens. Secondly, 3 copies of normal tissues, lesions and tissues around them are taken within 30 minutes; 3 copies of the specimens at the lesion are taken if it is a single focus; make them aliquots in freezing tubes with patient's information. Thirdly, the specimens are placed in net bags at corresponding marked positions, and they are put in a liquid nitrogen tank at $-196^{\circ} \mathrm{C}$ for rapid freezing. Fourthly, move it to a refrigerator at $-80^{\circ} \mathrm{C}$ for storage and standby after taking it out. Lastly, all relevant information of the specimen is registered and it is uploaded to the database after completing the above operations. Corresponding photos are needed to be uploaded before and after taking the specimen.

\subsubsection{Blood samples}

Whole blood is needed to be collected with 3 mlEDTA anticoagulation tube. The operator should mix the samples upside down several times after collection, and make them aliquots into three freezing tubes respectively. $5 \mathrm{ml}$ heparin sodium anticoagulation tube is used to collect plasma. After collecting blood, operator need to mix it upside down and let it stand to prevent hemolysis; operator uses centrifuge to separate plasma within 2 hours and sampler to absorb the separated plasma, and they packs it at $500 \mu \mathrm{l} /$ tube under aseptic conditions. Blood serum was collected by coagulation promoting vessels, and serum is collected after being centrifuged and discarded other blood clots. The specimens need to be marked and numbered after processing, and stored in a refrigerator at $-80^{\circ} \mathrm{C}^{[2]}$.

\subsubsection{Paraffin specimens}

Firstly, the pathological specimens are fixed with $10 \%$ formalin within 48 hours. Then, three lesions, three adjacent tissues and three normal tissues are taken, dehydrated, stained and embedded according to the conventional pathological techniques, and they are made into paraffin blocks to store in the specimen bank.

\subsection{Marking and storage of specimens}

To ensure the uniqueness of specimen coding, the format of specimen coding is area zip code-patient hospitalization number-year-specimen type-No.N. After printing the barcode, operators need to tick it on the freezing tube cover or wall and put it into the freezing box in sequence, and they record the position of each specimen in the freezing box, freezing warehouse and refrigerator. Besides, they also need to store the specimen record data item by item and upload it, and they call up the specific information of the specimen by scanning the barcode ${ }^{[3]}$.

\subsection{Information management}

A data management software is introduced to manage the digital data of biological sample database, which includes basic information, such as specimen storage label, type, storage time, sampling location and storage method, specification, number, storage equipment number, sample number and storage coordinates of the freezing box, outbound label, date, user, user, transferred-out quantity, and original storage location of samples. In addition, the database also includes comprehensive query functions, such as data import, statistics and query. Specimens should be registered by special personnel when entering or taking from the warehouse. Users can only use them after the application submitted to the ethics committee is approved.

\section{Results}

Currently, there are more than 20,000 specimens of various benign and malignant cases are collected in the biobank in our hospital, which covers 380 diseases, including paraffin-embedded tissue, fresh frozen tissue, femoral head, whole blood, plasma, serum, cerebrospinal fluid, etc. 10-15 pieces of paraffin tissue and frozen tissue are randomly sampled regularly for HE staining, 
DNA, RNA, immunohistochemistry, protein and other quality tests to ensure the storage quality of specimens. A large number of these beneficial specimens are used in clinical research at present.

\section{Discussion}

In recent years, the concept of translational medicine has been put forward in the international medicine and health filed. It means a continuous process from experimental platform to clinic. The concept has been put widely attentions by the international medical community once it been put forward. It bridges the gap between clinic and scientific research, and can guide the basic research results by laboratories to clinical medical applications more quickly, which improves the level of disease diagnosis, treatment and prevention ${ }^{[4]}$. The purpose of developing a biobank in a general hospital is to make more effective and rational use of human specimen. Guided by translational medicine, scientific research will be carried out on clinical specimens, and the results will be used for clinical services, breaking the barrier between clinical application and medical basic research and technology development ${ }^{[5]}$.

Biobank needs to meet the relevant standards of international organizations. Strict specimen collection procedures and standardized storage methods are the basic prerequisites for clinical scientific research and external resource sharing. The quality of specimen collection and storage is related to the reliability of scientific research results. The experimental quality can be effected by improper collection and storage, contamination of specimens, wrong sampling position, protein degradation, etc., resulting in non-accurate results and bringing troubles to scientific research work. Our hospital has been exploring a scientific and standardized management mode, and has summed up a feasible specimen collection processes. A set of standardized operation procedures, such as a process of collection of samples from tissues to blood and body fluids, sampling, storage and use of self-operated specimens in vitro, the numbering information management of specimen data, regular sampling of sample quality, has been set up to avoid the degradation of RNA, DNA and protein, and to ensure the integrity and quality of specimen storage. Currently, there are more than 20,000 specimens being collected in the biobank in our hospital, most of which are being used for scientific researches. However, except for the standardized management process to make full use of specimens, there are still ethical challenges when biological sample databases are constructing.

Different from the standard clinical trials of drugs, the ethical review of biobank is of particularity. There are five ethical challenges, including informed consent, trust, confidentiality, cooperation and commercialization throughout the construction of biobank. Generally speaking, the source of biological samples comes from large-scale collection based on a certain purpose or natural collecting accumulation. The common channels for biological samples mainly include surgical diagnosis and treatment, case examination, medical examination, etc. Therefore, ethical issues are also involved, such as informed consent of sample sources, privacy protection of sample management, etc. It can be seen that the ethical challenges continue throughout the whole process of sample generation, collection and use in constructing biobanks. Relevant investigations show that there are plenty of problems faced by hospital ethics committees during the construction and management of biobanks in China, such as insufficient legal support, unreasonable institutional setup, lack of ethical training, etc. How to balance the relationship between the protection and sharing of biological sample banks is an important problem to be solved urgently. Due to the influence of national conditions, legal and ethical development and the biological database construction in current situation, it is difficult to absolutely protect the privacy of participants in practice. A lot of private information of sample source can be found out through DNA, and the measures of de-identification become a mere formality. Therefore, preparation and prediction work should be done in the early stage of biological sample construction. Strict management procedures should be established in sample collection, ethical review, informed consent, specimen processing, storage, annotation, use, transportation and destruction. Measures, such as an accountability and punishment system, restriction of sample access, should be promoted to strengthen privacy protection ${ }^{[6]}$.

In summary, the development of biobank is of great significance for clinical treatment and researches in life science. Our hospital, based on the achievements of biobank, is further improving the way of resource utiliza- 
tion. However, there are still many ethical risks ahead of biobank. Except for having a forward-looking vision, an ethical worker should notice potential ethical risks and regulate self-behavior to control risks. He or she needs to protect the rights and interests of sample providers and promote the sustainable development of biobank.

\section{Acknowledgement}

This article was supported by Science and Technology Projects of Baoding (No. 2041ZF163).

\section{References}

1. Cheng M, Feng J. Management of biosamples transferred from hospitals to laboratories: Main problems and solutions. Chinese Journal of Tissue Engineering Research 2018; 22(16): 2619-2624.
2. Li Y, Ding Q, Wang X, et al. Establishment and management of Shaoxing cancer biobank (in Chinese). Zhejiang Medical Journal 2018; 40(16): 1850-1854.

3. Yang J. Preservation of pathological archives and realization of the value of its biological sample database attributes (in Chinese). Chinese Medicinal Biotechnology 2018; 13(5): 474-476.

4. Wu C, Lei R. Ethical and legal thinking on integration of human biobank and health information database. Studies in Dialectics of Nature 2019; 35(10): 63-68.

5. Zhang Y, Yang Q, Zhang H, et al. A novel vitrified cryopreservation method for the establishment of tumor tissue biobank. Chinese Journal of Tissue Engineering Research 2018; 22(4): 587-592.

6. Zhang Q, Liu W, Wang Z, et al. Construction of Biobank based on the real world study. Chinese Journal of Medical Science Research Management 2019; 32(6): 423-426. 